\title{
Chemical Shift and Electric Field Gradient Tensors for the Amide and Carboxyl Hydrogens in the Model Peptide $N$-Acetyl-D,L-valine. Single-Crystal Deuterium NMR Study
}

\author{
Rex Gerald II, ${ }^{\dagger}$ Thomas Bernhard, ${ }^{\ddagger}$ Ulrich Haeberlen, ${ }^{*}$ John Rendell, ${ }^{8}$ and \\ Stanley Opella
}

Contribution from the Department of Chemistry, University of Illinois at Chicago, Chicago, Illinois 60680, Arbeitsgruppe Molekülkristalle, Max-Planck-Institut für Medizinische Forschung, 6900 Heidelberg, Germany, and Department of Chemistry, University of Pennsylvania, Philadelphia, Pennsylvania 19104. Received July 27, 1992

\begin{abstract}
Single-crystal samples of the peptide $N$-acetyl-D,L-valine (NAV) with the exchangeable amide and carboxyl hydrogens replaced by deuterons were investigated by deuterium NMR spectroscopy. The electric field gradient (EFG) and chemical shift (CS) tensors for the amide and the carboxyl hydrogens, both of which are involved in intermolecular hydrogen bonds, were determined. The relationship of these tensors to the structure of NAV is discussed. The orientations of the EFG and the CS tensors of the amide hydrogen in a protein can provide information about the polypeptide backbone structure. For NAV the eigenvector of the largest eigenvalue of the EFG tensor coincides with the $\overrightarrow{\mathrm{NH}}$ bond direction, as found by $\mathrm{X}$-ray crystallography, within $2^{\circ}$, and the eigenvector of the intermediate eigenvalue with the normal $\left(\vec{n}_{\text {peptide }}\right)$ to the peptide plane within $1^{\circ}$. The directions of largest and smallest shielding are similarly aligned with $\overrightarrow{\mathrm{NH}}$ and $\vec{n}_{\text {peptide, }}$ respectively. However, the deviations are considerably larger, namely $9^{\circ}$ and $11^{\circ}$, respectively. The anisotropy of the CS tensor is determined to be $13.4 \pm 2.7 \mathrm{ppm}$, confirming that the amide hydrogen is involved in a weak hydrogen bond.
\end{abstract}

\section{Introduction}

Solid-state NMR spectroscopy is well established as a method for describing molecular structure with resolution on the atomic scale. Many of the NMR observables result from anisotropic interactions between the nuclear spin and its environment. These observables can be described by second-rank tensors. For example, the eigenvalues of the traceless symmetric part of the hydrogen chemical shift (CS) tensor provide information about the strength of inter- or intramolecular hydrogen bonding. ${ }^{1}$ On the other hand, the eigenvectors of the deuterium electric field gradient (EFG) tensor give deuteron/proton bond directions with an accuracy rivalled only by neutron diffraction.

In this paper we report structural information of this type for the amide and carboxyl hydrogen sites in a single crystal of the model peptide $N$-acetyl-D,L-valine (NAV); see Figure 1 . We use deuterium NMR to infer both the EFG and the CS tensors at the amide and carboxyl hydrogen sites in NAV. Advantages of this technique over multiple-pulse proton NMR are that it works in the presence of ${ }^{14} \mathrm{~N}$ spins which are very hard to decouple from protons and that additional information in form of the EFG tensors can be derived. The change in the CS and EFG tensors upon exchange of a deuteron for a proton (the isotope effect) is anticipated to be very small; the effect on the CS tensors is certainly smaller than the experimental errors.

NAV has served as a model peptide before in a variety of NMR studies, ${ }^{2-5}$ including those concerned with developing solid-state NMR spectroscopy as a method for determining the structure of proteins. NMR experiments on peptide or protein samples which are oriented in at least one dimension can provide important information about the three-dimensional structure of the peptide or the protein. ${ }^{6.7}$ In order to interpret the NMR data in terms of the structure of the polypeptide, the relationship of the CS and EFG tensors to the local symmetry elements of an amino acid, e.g., the peptide plane, is essential. The main purpose of this work is to investigate this relationship for the amide hydrogen CS tensor.

As far as we know, this is the first complete measurement of an amide hydrogen CS tensor. It allows the comparison with

\footnotetext{
-Address of corresponding author: Prof. U. Haeberlen, Max.Planck-Institute für Medizinische Forschung, Arbeitsgruppe Molekülkristalle, Jahnstrasse 29, 6900 Heidelberg, Germany.

+ University of Illinois at Chicago.

; Max-Planck-Institut far Medizinische Forschung.

University of Pennsylvania.
}

previous less complete data for acetanilide (AA) by Reimer et al. ${ }^{8}$ and with recent ab initio calculations for Glycylglycine (GG) by Chesnut et al.,9 see Figure 1.

The amide hydrogen CS tensor will also provide orientational information for peptide bonds in proteins complementary to that from the nitrogen CS and EFG tensors and the nitrogen-hydrogen heteronuclear dipole-dipole coupling which have been used previously to determine protein structures by solid-state NMR spectroscopy. ${ }^{7}$ This information will be particularly valuable because the amide hydrogen CS tensor is not axially symmetric. In addition, the use of the amide hydrogen CS interaction in high-field solid-state NMR experiments will increase the available resolution among peptide sites.

\section{Experimental Section}

NAV crystallizes in the monoclinic system, space group $P Z_{1} / c$ (no. 14 of ref 10); $a=6.626 \AA, b=13.002 \AA, c=10.031 \AA, \beta=105.37^{\circ} .11$ The unit cell is comprised of four molecules. Figure 2 shows the orientation of nine molecules in the crystal of NAV as determined X-ray crystallography." All molecules shown intersect the $c$-glide plane at $b / 4$. The two molecules omitted from the unit cell are related to the two shown via the inversion center at $[1 / 2,1 / 2,1 / 2]$ or, equivalently, via the two $2_{1}$ screw axes parallel to $\vec{b}$ that intersect the $a, c$-plane at $1 / 2 a+1 / 4 c$ and $1 / 2 a+3 / 4 c$. Note that each molecule is connected to four of its neighbors by hydrogen bonds and that the oxygen $\mathrm{O}_{1}$ is involved in two hydrogen bonds. The crystals used in this study were deuterated at both the carboxyl and the amide positions, such that all hydrogen bonds are formed by deuterons rather than protons. Since molecules related by

(1) Berglund, B.; Vaughan, R. W. J. Chem. Phys. 1980, 73, 2037-2043.

(2) Stark, R. E.; Haberkorn, R. A.; Griffin, R. G. J. Chem. Phys. 1978, 68, 1996-2001.

(3) Stark, R. E.; Jelinski, L. E.; Ruben, D. J.; Torchia, D. A.; Griffin, R. G. J. Magn. Reson. 1983, 55, 266-272.

(4) Tycko, R.; Stewart, P. L.; Opella, S. J. J. Am. Chem. Soc. 1986, 108, 5419-5425.

(5) Ramanathan, K. V.; Opella, S. J. J. Magn. Reson. 1988, 78, 367-370.

(6) Stewart, P. L.; Tycko, R.; Opella, S. J. J. Chem. Soc., Faraday Trans. 1 1988, 84, 3803-3819.

(7) Opella, S. J.; Stewart, P. L. Methods Enzymol. 1989, 176, 242-275.

(8) Reimer, J. A.; Vaughan, R. W. J. Magn. Reson. 1980, 41, 483-491.

(9) Chesnut, D. B.: Phung, C. G. Chem. Phys. Lett. 1991, 183, 505-509. Chesnut, D. B.; Phung, C. G. In The Calculation of NMR Shielding Constants; Tossell, F. A., Ed.; Kluwer Academic Publishers: Norwell, MA, 1993. (10) International Tables for Crystallography; Hahn, T., Ed.; D. Reidel Publishing Company: Dordrecht, The Netherlands, 1983.

(11) Carroll, P. J.; Stewart, P. L.; Opella, S. J. Acta Crystallogr. 1990. C46, 243-246. 


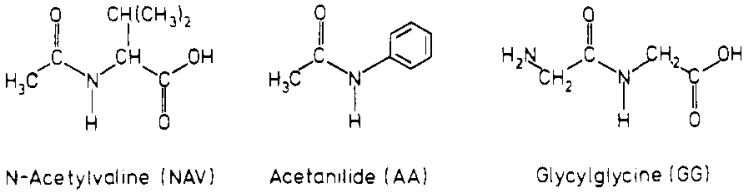

Figure 1. The three peptides NAV, AA, and GG.

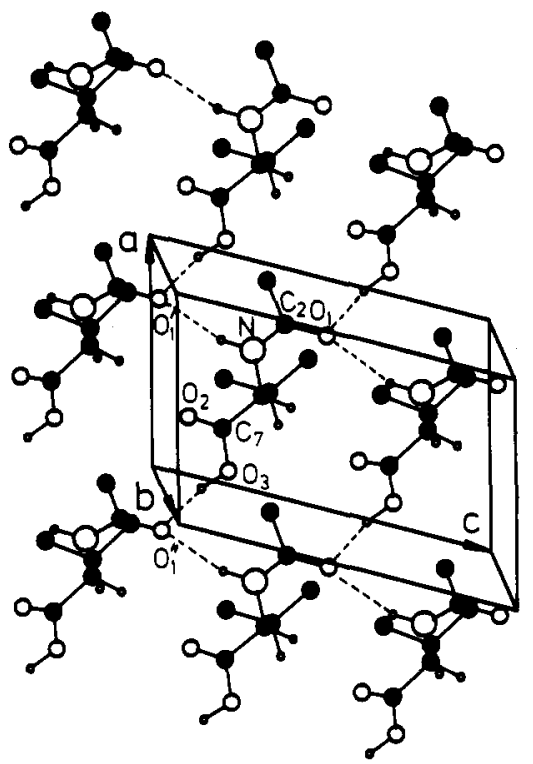

Figure 2. Arrangement of the NAV molecules as determined by X-ray crystallography. ${ }^{11}$ The black, the large white, the medium white, and the small white circles indicate respectively carbons, nitrogens, oxygens, and hydrogens. Hydrogens belonging to methyl groups are omitted. The dashed lines indicate hydrogen bonds. Only molecules that intersect the $c$-glide plane at $b / 4$ and, thus, only two of the four molecules in the depicted unit cell are shown.

inversion yield the same NMR resonances, there are only two magnetically nonequivalent molecules per unit cell. The EFG and CS tensors of the carboxyl and amide deuterons of these two molecules are related by the same symmetry operations as the molecules themselves, i.e. by the c-glide plane or, equivalently, by the 2, screw axes. Thus, the tensors of the two symmetry-related molecules have the same eigenvalues and differ only in the orientation of their eigenvectors.

Two crystals of NAV grown from $D_{2} O$ solution (one about $70 \mathrm{~mm}^{3}$ the other $40 \mathrm{~mm}^{3}$ ) were oriented using an optical two-circle goniometer and glued to PVC rods in such a way that they could be rotated, one about the crystal $c$-axis, and the other about the axis given by $\hat{a}^{*}+\hat{b}^{*}$, where " ${ }^{*}$ denotes a unit vector and "*n a reciprocal lattice vector. These two crystals will be referred to as the $c$-crystal and the $\left(a^{*}+b^{*}\right)$-crystal in what follows. Due to the presence of the $2_{1}$ screw axis symmetry element, the latter yields identical information to that obtained from a crystal which is rotated about the axis $-\hat{a}^{*}+\hat{b}^{*}$.

The crystal $c$-axis was chosen as a rotation axis because it is unique and easily identified as being parallel to a set of edges of the macroscopic crystal. The $2_{1}$ screw axis transforms $\vec{c}$ into its negative. On the other hand, the direction of the second rotation axis is transformed by the 2 , screw operation into a third virtual rotation axis given by $-\hat{a}^{*}+\hat{b}^{*}$. Thus, by choosing $\hat{a}^{*}+\hat{b}^{*}$ as a second rotation axis we obtain altogether rotation patterns for three different axes.

In the NMR goniometer, the rotation axis is perpendicular to the applied field, $\vec{B}_{0}$. Deuteron NMR spectra were recorded in a field of 8.4 $T$ by Fourier transforming the free induction decay (FID) following a radiofrequency pulse at $54.7 \mathrm{MHz}$. The pulse duration was $3.5 \mu \mathrm{s}$, corresponding to a flip angle of the deuteron magnetization of about $\pi / 2$. The longitudinal relaxation time, $T_{1}$, was measured and found to be about $30 \mathrm{~s}$. A relaxation delay of $180 \mathrm{~s}$ was used between $\pi / 2$ pulses during signal accumulation.

\section{Determination of the EFG/QC and CS Tensors}

Each magnetically nonequivalent deuteron yields one pair of lines in the ${ }^{2} \mathrm{H}$-spectrum of a single crystal. Its splitting is given by

$$
\Delta \nu=V_{z z}
$$

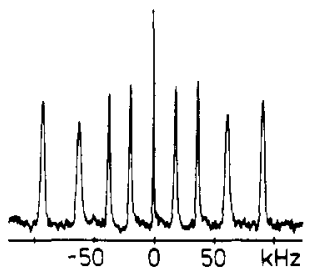

Figure 3. Typical ${ }^{2} \mathrm{H}$-NMR spectrum of the $c$-crystal. The sharp line in the center is caused by fluid $\mathrm{D}_{2} \mathrm{O}$ occluded in the crystal. The outer two line pairs belong to amide the others to carboxyl deuterons.

where $V_{z z}$ is the $z z$-component of the quadrupole coupling (QC) tensor, $\vec{V}$, in the laboratory frame, defined by $z \| \vec{B}_{0}$. The QC tensor and the EFG tensor, $\tilde{\Phi}$, are related by

$$
\tilde{V}=\frac{3}{2} \frac{e Q}{h} \Phi
$$

where $e, h$, and $Q$ are the fundamental charge, Planck's constant, and the quadrupole moment of the deuteron, respectively.

The center of gravity of the pair of lines measured relative to the Larmor frequency of a reference deuteron represents the chemical shift of the observed deuteron plus the so called second-order quadrupole shift. If the latter is subtracted from the center of gravity, the resulting frequency $\nu$ is given by

$$
\nu=\sigma_{z z} \nu_{\text {Larmor }}
$$

where $\sigma_{z z}$ is the $z z$-component of the CS tensor, $\tilde{\sigma}$, in the laboratory frame. The resonance frequency of TMS is typically taken as the reference frequency. $\tilde{\sigma}$ can be written as the sum of an isotropic part and a traceless symmetric tensor, $\tilde{\sigma}^{\text {ts. }}$. The order of magnitude of the eigenvalues of $\tilde{\sigma}^{\text {ts }}$ is $10 \mathrm{ppm}$, corresponding to $550 \mathrm{~Hz}$ at $8.4 \mathrm{~T}$. The maximum shift variation on reorienting the crystal is thus much smaller than the maximum quadrupolar splitting, which is roughly $250 \mathrm{kHz}$. Therefore, a meaningful measurement of a CS tensor requires a much more accurate determination of the line positions in the ${ }^{2} \mathrm{H}$-spectra than the measurement of a QC tensor (see below).

Since there are two magnetically nonequivalent NAV molecules in the unit cell, we observe four pairs of lines in the ${ }^{2} \mathrm{H}$-spectrum for an arbitrary crystal orientation, as can be seen in the spectrum shown in Figure 3. The sharp line at the center is caused by fluid $\mathrm{D}_{2} \mathrm{O}$ occluded in the crystal.

In order to determine the QC and CS tensors, we recorded rotation patterns of the line positions for the two differently mounted crystals at room temperature. The crystals were rotated in increments of $10^{\circ}$ about their respective rotation axes, and a spectrum was recorded for each crystal orientation. For the determination of the QC tensors, the positions of the lines, i.e. their centers of gravity, were estimated directly from the spectra. The variation of the line positions with the rotation angle $\varphi$ for the two crystals thus obtained is shown in Figure 4. Sine curves of the form

$$
\nu(\varphi)=\nu_{0}+a \cos \left[2\left(\varphi-\varphi_{0}\right)\right]
$$

were fitted to the data. They are also depicted in Figure 4. It is straightforward to decide which two curves correspond to the amide and which to the carboxyl deuterons, since the amide deuteron resonances are broadened by dipolar coupling to the directly bonded ${ }^{14} \mathrm{~N}$-nucleus. In fact at rotation angles corresponding to the largest coupling, dipolar splittings of the deuteron resonances are observable, as shown in Figure 5. Thus we can clearly distinguish the amide and carboxyl deuteron resonances. The solid curves in Figure 4 correspond to the amide deuterons, the dashed ones to the carboxyl deuterons.

The QC tensors are determined by least squares fitting of the line splittings corresponding to the data displayed in Figure 4 to symmetrical, traceless second-rank tensors. The rotation pattern from the $\left(a^{*}+b^{*}\right)$-crystal contains information for two rotation axes, $\hat{a}^{*}+\hat{b}^{*}$ and $-\hat{a}^{*}+\hat{b}^{*}$. We elaborate this point for the amide deuterons. One amide $Q C$ tensor is obtained by assigning the sine curves 1 and $1^{\prime}$ in Figure $4 a$ to the rotation axis $\hat{a}^{*}+\hat{b}^{*}$ and the 

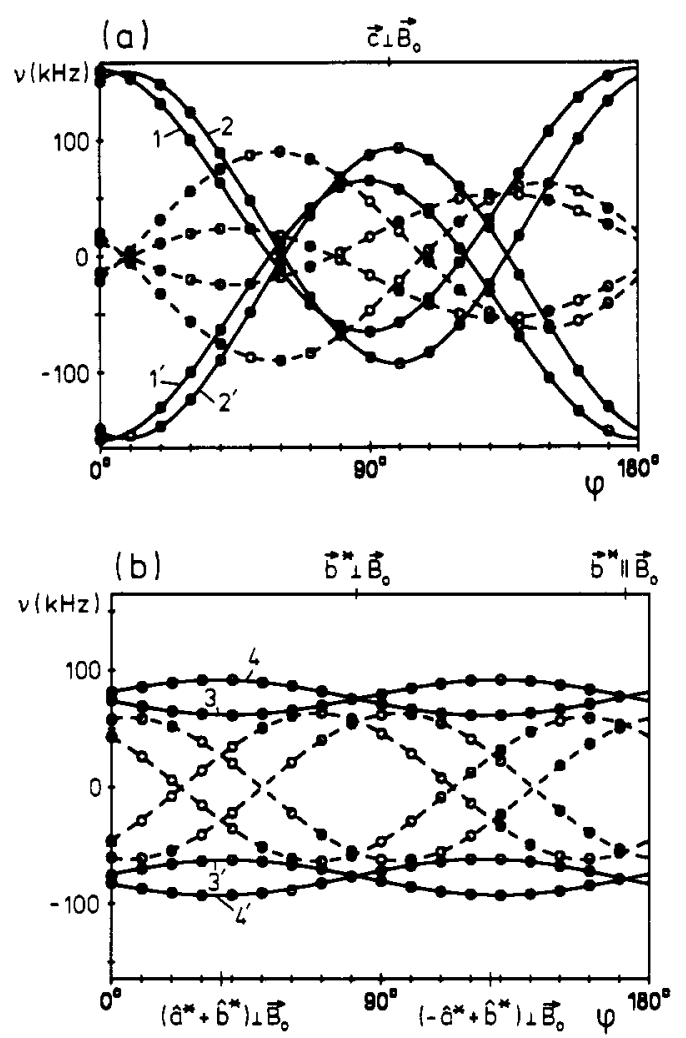

Figure 4. Rotation patterns of the line positions for (a) the $\left(a^{*}+\right.$ $b^{*}$ )-crystal and (b) the $c$-crystal. The sine curves are least squares fits to the data points. The solid ones refer to amide, the dashed ones, to carboxyl deuterons. The rotation angles at which the rotation patterns intersect are marked. The intersection occurs for $\vec{c} \perp \vec{B}_{0}$ in part a and $\left(\hat{a}^{*}+\hat{b}^{*}\right) \perp \vec{B}_{0}$ and $\left(-\hat{a}^{*}+\hat{b}^{*}\right) \perp \vec{B}_{0}$ in part b. In part $\mathrm{b}$ the rotation angles that refer to $\vec{b}^{*} \perp \vec{B}_{0}$ and $\vec{b}^{*} \| \vec{B}_{0}$ are also indicated. Since there are $2_{1}$ screw axes parallel to $\vec{b}$, the rotation pattern (b) is symmetric with respect to these two rotation angles. Note that $\vec{b}^{*}$ and $\vec{b}$ are identical.

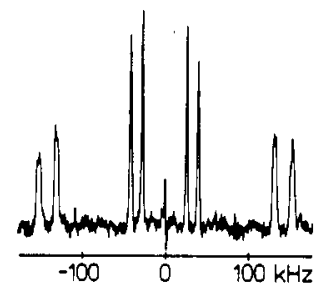

Figure 5. ${ }^{2} \mathrm{H}-\mathrm{NMR}$ spectrum of the $\left(a^{*}+b^{*}\right)$-crystal with conspicuous dipolar splittings of the amide deuteron lines (outer two line pairs).

curves 2 and $2^{\prime}$ tc $-\hat{a}^{*}+\hat{b}^{*}$. The corresponding curves in the rotation pattern of the $c$-crystal are identified by considering the intersection of the rotation patterns from the two crystals. The intersection is where the orientation of $\vec{B}_{0}$ is the same for both crystals, i.e. where $\left(\hat{a}^{*}+\hat{b}^{*}\right) \perp \vec{B}_{0}$ or $\left(-\hat{a}^{*}+\hat{b}^{*}\right) \perp \vec{B}_{0}$ for the $c$-crystal and $c \perp \vec{B}_{0}$ for the $\left(a^{*}+b^{*}\right)$-crystal. At the intersection the line positions must be the same in the two rotation patterns. The respective rotation angles are marked in Figure 4 . The consideration of the splittings at these orientations makes clear that curves 3 and $3^{\prime}$ from the c-rotation pattern (Figure $4 \mathrm{~b}$ ) must be assigned to the curves 1 and $1^{\prime}$ for rotation about $\hat{a}^{*}+\hat{b}^{*}$ and to 2 and $2^{\prime}$ for rotation about $-\hat{a}^{*}+\hat{b}^{*}$ to yield one of the amide deuteron QC tensors. The opposite assignment, i.e. curves 1 and $1^{\prime}$ to $-\hat{a}^{*}+\hat{b}^{*}, 2$ and $2^{\prime}$ to $\hat{a}^{*}+\hat{b}^{*}$, and 4 and $4^{\prime}$ to $\vec{c}$, yields the QC tensor of the second amide deuteron.

The two amide deuteron QC tensors thus obtained must be symmetry related by the 2, screw axis. Indeed we find that their eigenvalues agree within $0.5 \mathrm{kHz}$. Also, after transforming one of the two tensors by a $C_{2}$-rotation about $\vec{b}$, the eigenvectors agree within $0.5^{\circ}$. The QC tensor listed in Table I was obtained by transforming one original $\mathrm{QC}$ tensor by such a $C_{2}$-rotation and
Table I. Deuteron Quadruple Coupling Tensors

\begin{tabular}{|c|c|c|c|c|}
\hline \multirow[b]{2}{*}{ site } & \multirow[b]{2}{*}{ eigenvalues $(\mathrm{kHz}$} & & \multicolumn{2}{|c|}{ eigenvectors $^{a}$} \\
\hline & & & $\theta(\mathrm{deg})$ & $\Phi(\operatorname{deg})$ \\
\hline $\mathrm{D}(\mathrm{N})$ & $\begin{array}{l}-186.5 \\
-132.7 \\
319.2 \\
\mathrm{QCC}^{b}=2\end{array}$ & $\begin{array}{r}\vec{e}_{X}{ }_{X C, N} \\
\vec{e}_{Y}{ }^{Q C, N} \\
\vec{e}_{Z}^{Q C, N} \\
2.6 \mathrm{kHz},\end{array}$ & $\begin{array}{r}82.0 \\
109.1 \\
20.9 \\
169\end{array}$ & $\begin{array}{r}49.5 \\
136.7 \\
161.0\end{array}$ \\
\hline $\mathrm{D}\left(\mathrm{O}_{3}\right)$ & $\begin{array}{r}-132.1 \\
-108.3 \\
240.4 \\
\text { QCC = }\end{array}$ & $\begin{array}{l}\vec{e}_{X}{ }^{\mathrm{QC}, \mathrm{O}} \\
\vec{e}_{Y}^{\mathrm{QC}, \mathrm{O}} \\
\vec{e}_{Z}^{\mathrm{QC}, \mathrm{O}} \\
.3 \mathrm{kHz},\end{array}$ & $\begin{array}{r}119.7 \\
64.4 \\
41.1 \\
01 .\end{array}$ & $\begin{array}{r}68.2 \\
142.3 \\
19.0\end{array}$ \\
\hline
\end{tabular}

${ }^{a}$ Polar angles $\theta$ and $\Phi$ in the standard orthonormal system (SOS); $x, y, z$ defined by $x\|a, z\| c^{*} .{ }^{b} \mathrm{QCC}=e Q \Phi_{Z z} / h=2 V_{Z z} / 3 .{ }^{c} \eta=\left(V_{Y Y}\right.$ $\left.-V_{X X}\right) / V_{z z}$

taking the average with the second one. This procedure cancels part of the error originating from imperfect crystal orientation.

The QC tensors of the carboxyl deuterons were determined in analogy to those of the amide deuterons from the carboxyl data shown in Figure 4. Again the QC tensor listed in Table I is the average of the two measured, symmetry-related tensors.

The CS tensors can be determined in very much the same way from rotation patterns of the centers of gravity of the line pairs for the two differently oriented crystals. The center of gravity of a pair of lines is the average of the centers of gravity of the two lines. Since the total variation of the shifts was about $20 \mathrm{ppm}$, the line positions had to be determined to within a few ppm (1 ppm corresponds to $55 \mathrm{~Hz}$ ). The widths of the carboxyl lines were about $1.5 \mathrm{kHz}$ for all crystal orientations. Due to the dipolar coupling to the directly bonded ${ }^{14} \mathrm{~N}$-nucleus, the widths of the amide deuteron resonances depend on the crystal orientation. The minimum width observed was about $2 \mathrm{kHz}$. For determining the centers of gravity of individual lines, we used a computer routine which considers the first moment of a line and is capable of finding its center of gravity within a fraction of the digital spectral resolution. The accuracy of the result increases with increasing signal/noise $(\mathrm{s} / \mathrm{n})$ ratio. For the spectra of the $c$-crystal that were recorded for determining the QC tensors, 50 scans were accumulated yielding a $\mathrm{s} / \mathrm{n}$ ratio of about 15 , which was plenty for the QC tensors but proved to be insufficient for determining the $\mathrm{CS}$ tensors. Therefore we recorded additional spectra from the $c$-crystal for seven selected rotation angles, using 200 scans $(10-\mathrm{h}$ measuring time) for each spectrum this time. Only the centers of gravity inferred from these seven spectra were used for the determination of the CS tensors.

For the $\left(a^{*}+b^{*}\right)$-crystal we attempted to achieve sufficient $\mathrm{s} / \mathrm{n}$ ratio from the beginning and thus sacrificed $24 \mathrm{~h}$ of spectrometer time for each spectrum of the rotation pattern (500 scans). The centers of gravity could, of course, only be obtained for nonoverlapping lines. For the amide deuterons the centers were only determined for lines that did not show dipolar splittings. This reduced the number of usable data points from the $\left(a^{*}+\right.$ $b^{*}$ )-crystal to 9 for each of the two amide deuterons and to 10 and 14 for the two carboxyl deuterons.

Next we corrected the data for the second-order quadrupole shift. Assuming axial symmetry of the corresponding QC tensors, this shift is given by ${ }^{12}$

$$
S^{(2)}(\Omega)=\frac{V_{Z Z}^{2}}{24 \nu_{\text {Larmor }}}\left[1-\frac{V_{z z}^{2}(\Omega)}{V_{Z Z}^{2}}\right]
$$

where $V_{z z}$ is the largest eigenvalue of the QC tensor and $V_{z z}(\Omega)$ the doublet splitting at the orientation $\Omega$ of the crystal. The data in Table $I$ indicate that the assumption of axial symmetry is reasonable in this case. Note that $S^{(2)}(\Omega)$ is always toward higher frequencies. The largest second-order shifts calculated in this way are $\sim 1.4 \mathrm{ppm}$ for the amide and $\sim 0.8 \mathrm{ppm}$ for the carboxyl deuterons. Thus they are considerably smaller than the maximum

(12) Abragam, A. The Principles of Nuclear Magnetism; Oxford University Press: London, 1961. 

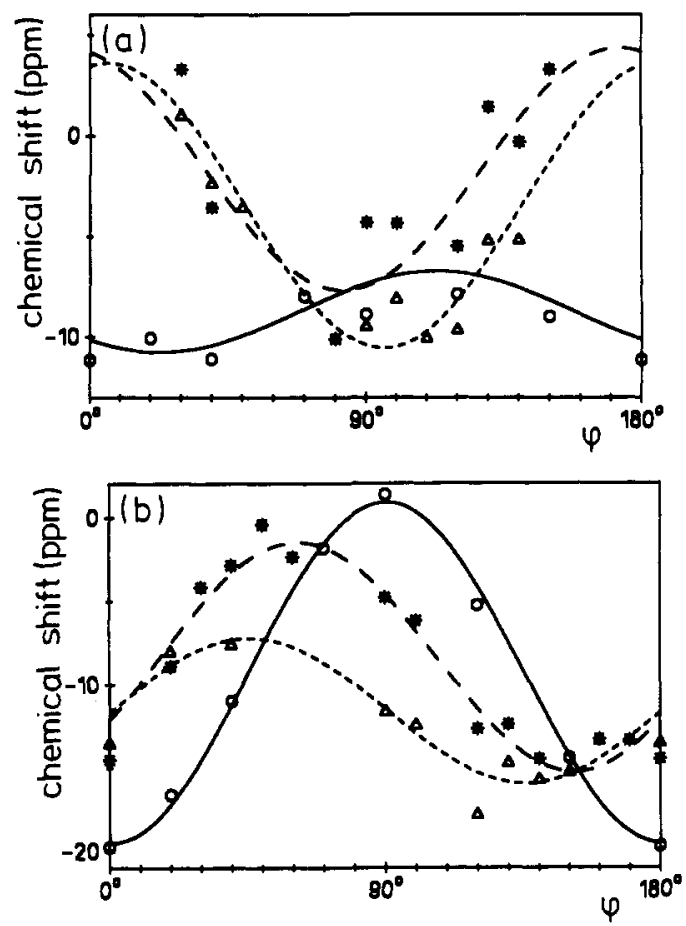

Figure 6. Rotation patterns of the chemical shift for (a) an amide and (b) a carboxyl deuteron. Circles and solid lines refer to the rotation axis $\vec{c}$, triangles and short dashed lines to $\hat{a}^{*}+\hat{b}^{*}$, and stars and long dashed lines to $-\hat{a}^{*}+\hat{b}^{*}$. The lines were calculated from the fitted CS tensors. The deviation of the points from the curves is somewhat larger for the amide than for the carboxyl deuteron. This is a consequence of the dipolar coupling of the amide deuteron to the directly bonded ${ }^{14} \mathrm{~N}$-nucleus complicating the accurate determination of the centers of gravity of the lines.

chemical shifts, yet they are not negligible. For all crystal orientations and line pairs that were used for the determination of the CS tensors, $S^{(2)}(\Omega)$ was calculated and subtracted from the measured shifts.

Before recording the rotation patterns, we adjusted the spectrometer frequency to the Larmor frequency of $\mathrm{D}_{2} \mathrm{O}$, intending to measure the CS tensors relative to $\mathrm{D}_{2} \mathrm{O}$ rather than to TMS. While the recording of the seven spectra from the c-crystal took only 3 days, the recording of the rotation pattern of the $\left(a^{*}+\right.$ $b^{*}$ )-crystal took about 3 weeks. The drift of our magnet is about $-3.2 \times 10^{-3} \mathrm{ppm} \mathrm{h}^{-1}$, leading to a drift of $1.6 \mathrm{ppm}$ in 3 weeks. Therefore, we also corrected the data from the $\left(a^{*}+b^{*}\right)$-crystal for the drift of the magnet.

The CS tensors were determined by least squares fitting of the shifts obtained in the way described above to symmetrical second-rank tensors. The assignment of the different data sets to different rotation axes was known from the determination of the QC tensors. Again, we get two CS tensors for the amide (carboxyl) deuterons which should be symmetry related by the 2, screw axis. The required symmetry was found to be fulfilled within 0.5 ppm with respect to the eigenvalues and within $1.5^{\circ}$ with respect to the eigenvectors.

The standard deviations of the data points from the shifts expected from the fitted tensors were $1.9 \mathrm{ppm}$ for the amide and $1.2 \mathrm{ppm}$ for the carboxyl deuterons. Therefore, we believe that the errors of the eigenvalues of the CS tensors are of this magnitude. The errors of the eigenvectors that belong to the largest eigenvalue are estimated to be $\pm 3^{\circ}$; those for the other eigenvectors might be somewhat larger.

Figure $6 a$ shows the rotation pattern of the shifts after the correction for the second-order quadrupole shift and the drift of the magnet for one of the two amide deuterons. Circles refer to the $c$-crystal and thus to the rotation axis $\vec{c}$; triangles and stars refer to the $\left(a^{*}+b^{*}\right)$-crystal and thus to the rotation axes $\hat{a}^{*}$ $+\hat{b}^{*}$ and $-\hat{a}^{*}+\hat{b}^{*}$. The solid curve shows the dependence of the shifts on the rotation angle $\varphi$ calculated from the fitted CS
Table II. Deuteron Chemical Shift Tensors

\begin{tabular}{|c|c|c|c|c|}
\hline \multirow[b]{2}{*}{ site } & \multirow[b]{2}{*}{ eigenvalues ${ }^{a}(\mathrm{ppm})$} & & \multicolumn{2}{|c|}{ eigenvectors ${ }^{b}$} \\
\hline & & & $\theta(\mathrm{deg})$ & $\Phi(\mathrm{deg})$ \\
\hline $\mathrm{D}(\mathrm{N})$ & $\begin{array}{c}-6.3 \\
-2.6 \\
8.9 \\
\sigma^{\text {iso } c=}=-9.3 \mathrm{ppm}\end{array}$ & $\begin{array}{l}\vec{e}_{X} \mathrm{CS}, \mathrm{N} \\
\vec{e}_{Y} \mathrm{CS,N} \\
\vec{e}_{Z} \mathrm{CS,N} \\
\sigma^{d}=13\end{array}$ & $\begin{array}{c}83.6 \\
117.6 \\
28.4 \\
, \eta^{e}=0.4\end{array}$ & $\begin{array}{r}59.2 \\
145.8 \\
161.3\end{array}$ \\
\hline $\mathrm{D}\left(\mathrm{O}_{3}\right)$ & $\begin{array}{c}-10.0 \\
-4.7 \\
14.7 \\
\sigma^{\text {iso }}=-14.5 \mathrm{ppm}\end{array}$ & $\begin{array}{l}\vec{e}_{X} \mathrm{CS}, 0 \\
\vec{e}_{Y} \mathrm{CS}, 0 \\
\vec{e}_{Z} \mathrm{CS}, 0 \\
\sigma=22 .\end{array}$ & $\begin{array}{c}95.4 \\
42.1 \\
48.5 \\
\eta=0.36\end{array}$ & $\begin{array}{r}96.8 \\
180.8 \\
11.7\end{array}$ \\
\hline
\end{tabular}

${ }^{a}$ Eigenvalues of the traceless part of $\tilde{\sigma} .{ }^{b}$ Polar angles in the SOS. ${ }^{c}$ Isotropic shift relative to TMS. ${ }^{d} \Delta \sigma=\sigma_{Z Z}-1 / 2\left(\sigma_{X X}+\sigma_{Y Y}\right)$. ${ }^{\prime} \eta=$ $\left(\sigma_{Y Y}-\sigma_{X X}\right) / \sigma_{Z Z}$.

tensor for rotation of a crystal about $\vec{c}$. The short dashed line shows this dependence for rotation about $\hat{a}^{*}+\hat{b}^{*}$ and the long dashed line for rotation about $-\hat{a}^{*}+\hat{b}^{*}$. Figure $6 \mathrm{~b}$ shows the corresponding data for one of the two carboxyl deuterons.

The resulting CS tensors are listed in Table II, taking into account the $4.8 \mathrm{ppm}$ difference in resonance frequency between TMS and water, TMS being more shielded. As before, the two tensors belonging to symmetry-related sites were averaged.

\section{Discussion}

4.1. Assignment of the Tensors to a Molecule in the Unit Cell. At this stage of the analysis we do not know to which of the two symmetry related but magnetically nonequivalent molecules in the unit cell the tensors stated in Tables I and II belong. This information cannot be extracted from our experimental data. However, it is well established that the unique eigenvector, $\vec{e}_{z}^{\circ}$, of a deuteron QC tensor is approximately parallel to the bond direction of the deuteron. ${ }^{13-19}$ For NAV the atomic positions have been determined by $\mathrm{X}$-ray crystallography. ${ }^{11}$ The angles between the eigenvectors $\vec{e} \mathrm{OC}$ of our QC tensors and the bond directions of the two symmetry-related deuterons are $1.8^{\circ}$ and $13.1^{\circ}$ for the amide and $6.5^{\circ}$ and $28.2^{\circ}$ for the carboxyl deuterons. Although it is true that the positions of hydrogens determined by $X$-ray crystallography are often somewhat unreliable, it is clear that the QC tensors listed in Table I must be assigned to the amide and the carboxyl deuterons giving angular deviations of $1.8^{\circ}$ and $6.5^{\circ}$, respectively. This immediately implies an unambiguous assignment also for the CS tensors, since those have been determined from the same sets of spectra as the QC tensors. We have prepared Tables I and II such that all listed tensors belong to the molecule with atomic positions $x, y$, and $z$ given in ref 11 .

4.2. Relationship between the QC and CS Tensors and the Molecular Structure. The eigenvector $\vec{e}_{2}{ }^{C}, \mathrm{O}$ of the carboxyl QC tensor deviates by as much as $6.5^{\circ}$ from the $\overrightarrow{\mathrm{O}_{3} \mathrm{H}}$ bond direction determined from X-ray analysis but only by $2.4^{\circ}$ from the $\mathrm{O}_{3} \mathrm{O}_{1}^{\prime \prime}$ interconnecting vector. For the labeling of the atoms, see Figure 2. We interpret this as an indication that the atoms in the $\mathrm{O}_{3}-\mathrm{H} \ldots \mathrm{O}_{1}{ }^{\prime \prime}$ hydrogen bond are more linearly arranged than is indicated by the X-ray crystal structure. $\vec{e}_{X}^{\mathrm{QC} . O}$ is approximately parallel to the normal, $\vec{n}_{\text {carboxyl, }}$ of the carboxyl plane defined by $\mathrm{O}_{2}, \mathrm{C}_{7}$, and $\mathrm{O}_{3}\left(\angle\left(\vec{e}_{X}^{\circ}, \delta_{,}, \vec{n}_{\text {carboxyl }}\right)=4.2^{\circ}\right)$, in agreement with previous measurements of deuterium QC tensors in comparable compounds. ${ }^{15}$ The small quadrupole coupling constant, QCC = $160.3 \mathrm{kHz}$, indicates a moderately strong hydrogen bond, con-

(13) Chiba, T. J. Chem. Phys. 1964, 39, 1352-1358.

(14) Soda, G.; Chiba, T. J. Chem. Phys. 1969, 50, 439-455.

(15) Blinc, R. In The Hydrogen Bond; Schuster, P., Zundel, G., Sandorfy, C., Eds.; North-Holland Publishing Company: Amsterdam, The Netherlands, 1976; Vol. II, pp 831-887.

(16) Müller, C.; Idziak, S.; Pislewski, N.; Haeberlen, U. J. Magn. Reson. 1982, 47, 227-239.

(17) Múller, C.; Schajor, W.; Zimmermann, H.; Haeberlen, U. J. Magn. Reson. 1984, S6, 235-246.

(18) Weeding, T.; Kwiram, A. L.; Rawlings, D. C.; Davidson, E. R. J. Chem. Phys. 1985, 82, 3516-3526.

(19) Slosarek, G.; Heuer, A.; Zimmermann, H.; Haeberlen, U. J. Phys.: Condens. Matter 1989, $1,5931-5954$ 
Table III. Experimental and Calculated Amide Hydrogen CS Tensor Components for Three Molecules

\begin{tabular}{|c|c|c|c|c|c|c|}
\hline molecule & $\sigma_{X X^{a}}^{a}$ & $\sigma_{Y Y}$ & $\sigma_{z z}$ & $\sigma^{\text {iso }}$ & $\Delta \sigma$ & $\overrightarrow{\mathrm{N} \cdots O}(\AA)$ \\
\hline $\begin{array}{l}\text { NAV }\left(\exp ^{b}\right) \\
\text { AA }\left(\exp ^{c}\right) \\
\text { GG }\left(\operatorname{calc}^{d}\right)\end{array}$ & $\begin{array}{l}14.9 \\
17.4 \\
12.6\end{array}$ & $\begin{array}{l}18.6 \\
17.5 \\
24.7\end{array}$ & $\begin{array}{l}30.1 \\
35.1 \\
38.3\end{array}$ & $\begin{array}{l}21.2 \\
23.3 \\
25.2\end{array}$ & $\begin{array}{l}13.4 \\
17.7 \\
19.7\end{array}$ & $\begin{array}{l}3.18 \\
2.97 \\
2.86\end{array}$ \\
\hline
\end{tabular}

${ }^{a}$ Absolute shielding values. Absolute shielding of TMS is $30.5 \mathrm{ppm} .{ }^{b}$ This study. ${ }^{C}$ Reimer and Vaughan. ${ }^{8}{ }^{d}$ Chesnut and Phung ${ }^{9}$.

sistent with the distance $\left|\overline{\mathrm{O}_{3} \mathrm{O}_{1}{ }^{\prime \prime}}\right|=2.62 \AA$, ${ }^{\prime \prime}$ although it deviates by $\sim 9 \%$ from the value predicted by the empirical formula ${ }^{20}$

$$
\mathrm{QCC}(\mathrm{kHz})=271-8.63 \times 10^{5} \exp (-3.48|\overrightarrow{\mathrm{OO}}|(\AA))
$$

An explanation for this rather large deviation might be that this formula is mainly based on data obtained from hydrogen bonds distinctly longer than the one considered here.

For the amide $Q C$ tensor, $e_{2}^{O C, N}$ deviates by only $1.8^{\circ}$ from the $\overrightarrow{\mathrm{NH}}$ bond direction established by $\mathrm{X}$-ray crystallography but by $20.0^{\circ}$ from $\overrightarrow{\mathrm{NO}_{1}{ }^{\prime}}$. This confirms the marked nonlinearity of the hydrogen bond in which the amide hydrogen is involved. $\vec{e}_{X}^{C, N}$ is nearly parallel to the normal, $\vec{n}_{\text {peptide, }}$ of the peptide plane defined by $N, C_{2}$, and $O_{1}\left(\angle\left(\vec{e}_{X}^{Q C}, N, \vec{n}_{\text {peptide }}\right)=0.9^{\circ}\right)$. Note that the asymmetry of the amide QC tensor is comparatively large $(\eta=0.169)$ and thus $\bar{e}_{X}^{\mathrm{OC}, \mathrm{N}}$ is particularly well defined. The large QCC (QCC $=212.6 \mathrm{kHz}$ ) is consistent with the weakness of the hydrogen bond $\left(\mid \overline{\mathrm{NO}_{1} \mid}=3.18 \AA\right)^{11}$ and agres within $3 \%$ with the value predicted by the empirical formula ${ }^{15}$

$$
\mathrm{QCC}(\mathrm{kHz})=253-\frac{512}{[R(\AA)]^{3}}
$$

where $R$ is the distance between $O_{1}{ }^{\prime}$ and D. Our NMR-QC-data thus confirm that the $\mathrm{N}-\mathrm{H} \cdots \mathrm{O}_{1}^{\prime}$ hydrogen bond is weak, asymmetric, and significantly nonlinear. On the other hand, we should also stress that our data are sensitive to these structural properties.

The eigenvectors of the CS tensors are less intimately related to the conformation of the NAV molecule compared to the eigenvectors of the QC tensors. For the carboxyl hydrogen, the direction of largest shielding, $\vec{e}_{Z}^{C S, O}$, deviates by $\sim 7^{\circ}$ when compared to the $\overline{\mathrm{O}_{3} \mathrm{O}_{1}}{ }^{\prime \prime}$ direction and by $\sim 15^{\circ}$ when compared to the $\overrightarrow{\mathrm{O}_{3} \mathrm{H}}$ direction. As we think that the $\overrightarrow{\mathrm{O}_{3} \mathrm{H}}$ direction is more reliably represented by $\vec{e}_{Z}^{O C, O}$, we should rather compare $\vec{e}_{Z}^{\mathrm{CS}, O}$ with $\vec{e}_{Z}^{C, O}$. The angle between these two directions is $\sim 9^{\circ}$. $\vec{e}_{X}^{C S, O}$ deviates by $\sim 40^{\circ}$ from $\vec{n}_{\text {carboxyl. }}$. The respective angles for the amide CS tensors are $\angle\left(\vec{e}_{Z}^{\mathrm{CS}, N}, \overrightarrow{\mathrm{NH}}\right) \approx 9^{\circ}, \angle\left(\vec{e}_{Z}^{\mathrm{CS}, N}, \overrightarrow{\mathrm{NO}_{1}}\right) \approx 19^{\circ}$, and $\angle\left(\vec{e}_{X}^{C S, N}, \vec{n}_{\text {peptide }}\right) \approx 11^{\circ}$. We attribute these angle deviations in part to the nonlinearity of the $\mathrm{N}-\mathrm{H} \ldots \mathrm{O}_{1}^{\prime}$ hydrogen bond and the lack of overall planarity of the molecule.

Figure 7 shows the orientations of the QC and the CS tensor of the amide hydrogen as compared to the molecular conformation.

4.3. Comparison of the CS Tensors with Other Experimental and Theoretical Data. The correlation between the length of $\mathrm{O}-\mathrm{H} \ldots \mathrm{O}$ hydrogen bonds and the isotropic chemical shift and the chemical shift anisotropy has been studied extensively, both experimentally and theoretically. ${ }^{1}$ Our data for the carboxyl hydrogen in NAV are in perfect agreement with previously published results.' The observation that the most shielded eigenvector is close to $\overline{\mathrm{O}_{3} \mathrm{O}_{1}{ }^{11}}$ is also consistent with previous studies. ${ }^{21,22}$

The value of the shielding anisotropy, $\Delta \sigma=13.4 \pm 2.7 \mathrm{ppm}$, places the amide hydrogen in NAV intermediate between hydrogens bonded to carbon and hydrogens involved in $\mathrm{O}-\mathrm{H}$... $\mathrm{O}$ bridges. The anisotropy of the former usually falls in the range 4-10 ppm, that of the latter in the range $15-30 \mathrm{ppm} .1,21,22$ The upper end of this range applies to strong, i.e. short, bridges. Our value is thus consistent with the fact that the amide hydrogen is

(20) Berglund, B.; Lindgren, J.; Tegenfeldt, J. J. Mol. Struct. 1978, 43, 179-191.

(21) Haeberlen, U. High Resolution NMR in Solids: Selective Averaging, Waugh, J. S., Ed.; Advances in Magnetic Resonance Supplement 1; Academic Press: New York, 1976.

(22) Mehring, M. Principles of High Resolution NMR in Solids, 2nd ed.; Springer: Berlin, 1983.
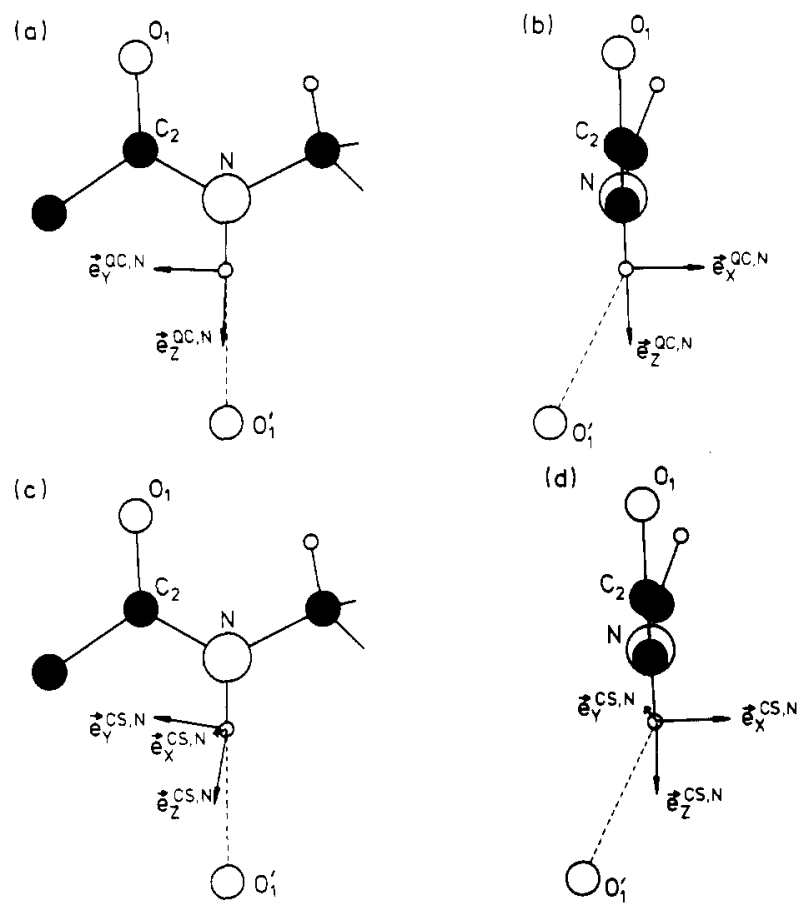

Figure 7. Orientation of the $\mathrm{QC}$ and the CS tensor of the amide hydrogen relative to the NAV molecule. For clarity only the amide group and its immediate neighborhood including the hydrogen-bonded oxygen $O_{1}{ }^{\prime}$ is shown. In parts a and $c$ the plane of the drawing is parallel and in parts $b$ and $d$ perpendicular to the peptide plane. The missing eigenvectors $\vec{e}_{X}^{C C, N}$ in part a and $\bar{e}_{Y}^{C}, N$ in part $b$ are hidden by the hydrogen atom.

involved in a rather weak hydrogen bond.

We know of only one other experimental attempt to determine the CS tensor of an amide hydrogen. Reimer and Vaughan applied a proton-selective multiple-pulse technique utilizing an ${ }^{15} \mathrm{~N}(100 \%)$ enriched powder sample of acetanilide (AA) (cf. Figure 1). ${ }^{8}$ We list their results in Table III together with the corresponding data of the present study of NAV. The larger values of $\sigma^{\text {iso }}$ and $\Delta \sigma$ in AA as compared to NAV are consistent with a more symmetric hydrogen bond in $\mathrm{AA}:|\overrightarrow{\mathrm{NO}}|=2.97 \AA$ in $\mathrm{AA},{ }^{8}$ $|\overrightarrow{\mathrm{NO}}|=3.18 \AA$ in NAV. ${ }^{11}$ Reimer and Vaughan were not able to determine the orientation of the most shielded direction of their almost axially symmetric CS tensor. Our results confirm their assumption that this direction is close to that of the NH bond. However, our results indicate that the deviation from axial symmetry of the amide CS tensor in AA is probably larger than that inferred by Reimer and Vaughan.

Recently, Chesnut and Phung have calculated the amide hydrogen CS tensor in glycylglycine (GG) (cf. Figure 1) by ab initio methods. ${ }^{9}$ They considered an isolated monomer and a monohydrate in two different conformations. In conformation I all heavy atoms were assumed to lie in a plane. Conformation II is obtained from I by a rotation through $90^{\circ}$ about the bond connecting the amide nitrogen with the methylene carbon, implying

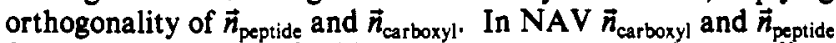
form an angle of $\sim 41^{\circ}$. Thus, NAV represents an intermediate case as compared to the two conformations of GG considered in the $a b$ initio calculations.

In the planar monomer, Chesnut and Phung find, not surprisingly, the largest shielding in the molecular plane. However, the variation of the shielding in this plane is small and the direction 
of largest shielding deviates by as much as $20^{\circ}$ from the amide bond. A second principal axis (the least shielded one) is dictated by symmetry to be perpendicular to the molecular plane. This symmetry is broken for the twisted form of GG. In both the monomer and the monohydrate twisted forms, the least shielded direction is calculated to deviate by $\sim 5^{\circ}$ from the normal of the peptide plane. Remember we find a deviation of $\sim 11^{\circ}$ between $\vec{e}_{X}^{C S, N}$ and $\vec{n}_{\text {peptide }}$ in NAV.

Upon hydration, the ab initio calculations yield an increase of $\Delta \sigma$ by $\sim 11 \mathrm{ppm}$ and a decrease of $\sigma^{\text {iso }}$ by $\sim 3 \mathrm{ppm}$. In Table III we list the results of the $a b$ initio calculation for the monohydrate in conformation I, for which a linear hydrogen bond between the amide nitrogen and the water oxygen and a distance $|\overrightarrow{\mathrm{NO}}|=2.859$ $\AA$ were assumed. The calculated numbers are in good agreement with the experimental results for AA and NAV. Note, the increase of $\sigma^{\text {iso }}$ and $\Delta \sigma$ with decreasing NO distance.

The $a b$ initio calculation for the planar monohydrate predicts the direction of largest shielding to be parallel to the $\mathrm{NH}$ bond. If a nonlinear hydrogen bond is assumed $\left(\angle(\overrightarrow{\mathrm{NO}}, \overrightarrow{\mathrm{NO}}) \approx 15^{\circ}\right)$, the direction of largest shielding is found to deviate from $\overrightarrow{\mathrm{NH}}$ by about $10^{\circ}$. For $\mathrm{NAV}$, where $\angle(\overrightarrow{\mathrm{NH}}, \overrightarrow{\mathrm{NO}}) \approx 20^{\circ}$, we measured a deviation between $\vec{e}_{Z}^{C S, N}$ and $\overrightarrow{N H}$ of $\sim 9^{\circ}$. Overall, the results of the $a b$ initio calculations are in good agreement with our experimental results.

\section{Conclusions}

We have shown that it is possible to measure the CS tensor of the amide hydrogen in the model peptide NAV by ${ }^{2} \mathrm{H}-\mathrm{NMR}$ on single-crystal samples. Our measurements also yield the CS tensor of the carboxyl hydrogen and the QC tensors of both the amide and carboxyl deuterons. All are in good agreement with previous experimental and theoretical results obtained from comparable compounds.

Our main purpose was to examine the relationship of the amide hydrogen CS tensor to the structure of the peptide. The CS tensor we obtained from the amide hydrogen in NAV is consistent with the results of a previous, but less complete, experimental study of acetanilide ${ }^{8}$ and the recent $a b$ initio calculations of glycylglycine. ${ }^{9}$ The results of our work and these two studies imply that there is a close correlation between the strength of the $\mathrm{N}-\mathrm{H}$.... hydrogen bond and the values of $\sigma^{\text {iso }}$ and $\Delta \sigma$. A similar correlation is well established for $\mathrm{O}-\mathrm{H} \ldots \mathrm{O}$ hydrogen bonds.

With respect to the orientation of the CS tensor of the amide hydrogen in NAV, we find that the most shielded eigenvector is close to the NH bond, while the least shielded is close to the normal of the peptide plane. The observed deviations of $9^{\circ}$ and $11^{\circ}$, respectively, are larger than the experimental error. We attribute these deviations to the lack of extended mirror symmetry in the NAV molecule (the dihedral angle between the peptide and the carboxyl plane is $\sim 41^{\circ}$ ) and to the nonlinearity of the $\mathrm{N}-\mathrm{H} \ldots \mathrm{O}$ hydrogen bond. The eigenvectors of the QC tensor of the amide deuteron are much more intimately related to the local molecular structure of the peptide than are those of the CS tensor: $\vec{e}_{2} \mathrm{C}, N$ deviates from $\overrightarrow{\mathrm{NH}}$ by only $1.8^{\circ}$, and $\vec{e}_{X}^{\mathrm{C}, N}$ from $\vec{n}_{\text {peptide }}$ by only $0.9^{\circ}$.

Acknowledgment. We thank A. Heuer for supplying computer fit routines, D. Theimer for his initial help in the measurements, and D. B. Chestnut for providing his CS tensor data for glycylglycine prior to publication. R.G. gratefully acknowledges the Max-Planck-Gesellschaft for support while in Heidelberg. J.R. was supported by a postdoctoral fellowship from the NRC of Canada. The research at the University of Pennsylvania was supported by Grants R01 GM-24266, R01 GM-29754, and R24 RR-05976 from the National Institutes of Health.

\title{
Electron Affinities, Enthalpy, and Entropy of Electron Attachment of Several $\eta^{4}$-(Olefin) $\mathrm{Fe}(\mathrm{CO})_{3}$ Complexes and Electron-Transfer Kinetics
}

\author{
Paul Sharpe and Paul Kebarle* \\ Contribution from the Department of Chemistry, University of Alberta, \\ Edmonton, Canada T6G 2G2. Received July 7, 1992
}

\begin{abstract}
The energies $\Delta H^{\circ}$ and $\Delta G^{\circ}{ }_{\mathrm{a}}$ and the entropy $\Delta S^{\circ}{ }_{\mathrm{a}}$ for the electron attachment (capture) process e $+\mathrm{B}=\mathrm{B}^{-}$were determined for the olefin iron tricarbonyls, where the olefin = 1,3-butadiene (Bd), 1,3-cyclohexadiene (CHD), 1,3,5-cycloheptatriene $(\mathrm{CHT})$, and cyclooctatetraene (COT). Data were obtained also for trimethylenemethane- $\mathrm{Fe}(\mathrm{CO})_{3}$ and cycloheptatriene- $\mathrm{Cr}(\mathrm{CO})_{3}$. The determinations were made with a pulsed electron high pressure mass spectrometer which allows measurement of electron-transfer kinetics and equilibria: $\mathrm{A}^{-}+\mathrm{B}=\mathrm{A}+\mathrm{B}^{-}$. Compounds $\mathrm{A}$ were reference compounds with known $\Delta H^{\circ}{ }_{\mathrm{a}}, \Delta G^{\circ}{ }_{\mathrm{a}}$, and $\Delta S^{\circ}{ }_{a}$. The thermodynamic and kinetic data indicate that for the $\mathrm{Ol}-\mathrm{Fe}(\mathrm{CO})_{3}$, electron capture leads to a reduction of hapticity from 4 to 2 for $\mathrm{Ol}=\mathrm{Bd}, \mathrm{CHD}, \mathrm{CHT}$ but a change over occurs for COT where the electron enters an orbital largely localized on the olefin.
\end{abstract}

\section{Introduction}

In previous work ${ }^{1,2}$ we reported measurements of the electron attachment (capture) free energy, $\Delta G^{\circ}{ }_{\text {, }}$, enthalpy, $\Delta H^{\circ}{ }_{a}$, and entropy, $\Delta S^{\circ}{ }_{\text {a }}$, involving $\eta^{4}-1,3$-butadiene iron tricarbonyl $\left(\mathrm{BdFe}(\mathrm{CO})_{3}\right)$. These relate to the gas phase reaction where $\mathrm{BdFe}(\mathrm{CO})_{3}=\mathrm{B}$.

$$
e+B=B^{-}
$$

(1) Dillow, G. W.; Nicol, G. Kebarle, P. J. Am. Chem. Soc. 1989, 111, 5465 .

(2) Dillow, G. W.; Kebarle, P. J. Am. Chem. Soc., in press.
The electron attachment energies and entropy were obtained from measurements of gas phase electron transfer equilibria 2 where $\mathrm{A}$ is a reference compound with known $\Delta G^{\circ}{ }_{\mathrm{a}}, \Delta H^{\circ}{ }_{\mathrm{a}}$, and $\Delta S^{\circ}{ }_{\mathrm{a}}$.

$$
\mathrm{A}^{-}+\mathrm{B}=\mathrm{A}+\mathrm{B}^{-}
$$

The equilibria 2 were measured with a pulsed electron high pressure mass spectrometer, PHPMS, using apparatus, techniques, and methodology which have been described in previous work. ${ }^{3,4}$

(3) Kebarle, P. In Techniques of Chemistry; Saunders, W. H., Farrar, J. M. Eds.; Wiley-Interscience: New York, 1988

(4) Kebarle, P.; Chowdhury, S. Chem. Rev. 1987, 87, 513. 